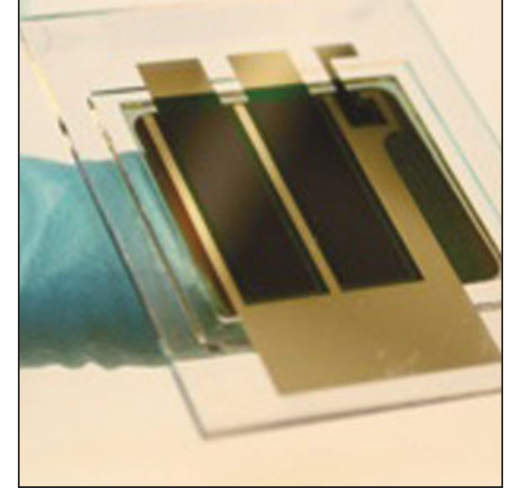

\section{Thinking small for solar}

\author{
By Corinna Wu \\ Feature Editor Hideyuki Murata
}

O n rooftops all over the world, silicon solar panels are harvesting sunlight and converting it into gigawatts of electricity. But now, many groups are hard at work developing solar technology that eschews silicon and other inorganic materials altogether. Organic photovoltaics (OPV), which uses organic materials such as polymers as the active component, follows on the heels of first-generation crystalline silicon and second-generation thin-film PV. OPV promises to be lightweight, flexible, and cheaper to manufacture. Its unique properties also open a range of applications not possible with inorganic solar materials.

Recently, new approaches to making OPV that use small organic molecules as the light-harvesting ingredient have posted some remarkable successes. Until now, it was unclear whether small-molecule OPV had the potential shown by polymer OPV technologies. But power conversion efficiencies reaching 10\% - near that of amorphous silicon-have encouraged companies and academic groups to plunge forward with their small-molecule OPV research.

Organic PV is attractive because the overall cost of the electrical power generated - expressed as lifetime multiplied by efficiency/manufacturing cost — can be extrapolated to become much lower than that of silicon or thin-film PV. The critical price point is much less than $\$ 1 / \mathrm{W}$, which is where "PV breaks even with some of the other conventional technologies that are used for power generation - namely, the burning of some kind of fossil fuel," said Bernard Kippelen, director of the Center for Organic Photonics and Electronics at the Georgia Institute of Technology.

The production cost of today's silicon PV technology is around $\$ 1-2 / \mathrm{W}$, Kippelen said, but the price of the raw material - pure, electronic-grade silicon - makes up a substantial portion of the cost of the device. "You can't go much beyond the cost of the materials that are needed to fabricate the cell unless someone comes up with a new process where you have a lowcost way to produce high-quality silicon," he said. "That would really require a breakthrough." The inorganic materials used in thin-film PV, such as cadmium telluride, have similar limitations for breakthrough raw material cost reductions.

On the other hand, organic molecules are typically cheaper and more abundant. The layers of materials in OPV can also be much thinner, so less material is needed. If a 100 -nm-thick layer can absorb sunlight effectively, the amount of material that coats $10 \mathrm{~m}^{2}$ would not be more than 1-2 grams, Kippelen said.

OPV can also be manufactured at low temperatures, which reduces the energy needed and allows for the use of plastic substrates. Common methods are spin-coating, die-coating, and organic vapor phase deposition onto a substrate. The energy payback for OPV can be measured in months, whereas for silicon, it typically takes two years for a silicon solar panel to generate enough energy to recover its manufacturing cost.

The ability to use plastic substrates also means that OPV can be lightweight and flexible - as can some second-generation thin-film inorganic cells. Structures might not need as much reinforcement to support the weight of such panels - all of which contribute to the overall cost.

For the past two decades, OPV researchers have been hard at work boosting efficiencies, an important component of the cost equation (see figure). The most advanced cells with organic molecules are the dye-sensitized ones, introduced by Michael Grätzel and Brian O'Regan at the École Polytechnique Fédérale de Lausanne, Switzerland, in 1991. Grätzel cells, as they are called, contain a thin layer of an organic dye to harvest sunlight and work, in principle, like an electrochemical cell to produce current. The best cell efficiency is greater than $12 \%$. However, the challenge for scaling up the technology and getting long operational lifetimes has been to make hermetic packages that seal in the electrolyte, Kippelen said.

For solid-state OPV, polymer-based solar materials currently dominate. Konarka in Lowell, Mass., has been ramping up its production, making polymer-based $\mathrm{PV}$ in sheets up to five feet wide. The company is also introducing PV in different colors to provide design options for architects, said Stuart Spitzer, Konarka's vice president of materials and engineering.

Polymers are easy to synthesize by bulk methods, but because the molecular weight and purity can be hard to control, the photovoltaic properties can also vary from one batch to the next. That is why some researchers have been taking a different tack by synthesizing small organic molecules for the sunlightabsorbing layer. "Once you have a particular batch of a small-

Hideyuki Murata, Japan Advanced Institute of Science and Technology

CorinnaWu, ckwu@nasw.org 
molecule material for solar cells, it will always behave that way, so your ability to properly manage the device fabrication will be a lot simpler," said Guillermo Bazan, an organic chemist at the University of California-Santa Barbara (UCSB).

But finding ways to make efficient solar cells from small molecules is a challenge. "When you cast from solution, you end up with a thin film. Usually small molecules do not give you good thin films. That's something that polymers do much better," Bazan said.

Recently, two companies working with small-molecule OPV have announced record-high efficiencies in prototype devices made from their materials. Researchers at the Mitsubishi Chemical Group in Japan have developed a solution-processable crystalline organic semiconductor that achieves 7.6\% efficiency. The material is tetrabenzoporphyrin (BP), a new "transformation semiconductor," according to Shinji Aramaki, senior researcher at Mitsubishi Chemical, who gave an overview of the group's work at the 2011 MRS Fall Meeting.

BP is highly crystalline, has high carrier mobility, and is insoluble, which makes working with it challenging, Aramaki said. However, a soluble precursor to BP can be spin-coated into an amorphous thin film that turns crystalline when heated.

The Mitsubishi Chemical Group improved upon their earlier work to achieve $10 \%$ efficiency in a single-junction device with an area of about $1 \mathrm{~cm}^{2}$ - a world OPV record. "Ten percent efficiency is an important milestone to prove that OPV is practical, because amorphous silicon PV's cell efficiency is around 10\%," Aramaki said. "Now we are more serious about the commercialization in Mitsubishi Chemical. We are planning the commercialization of OPV in 2015."

In December, the German company Heliatek announced its achievement of $9.8 \%$ efficiency in a $1.1 \mathrm{~cm}^{2}$ tandem cell - two solar cells stacked on top of one another-deposited on glass. The molecule they use is a thiophene derivative, which absorbs light in the $440 \mathrm{~nm}$ to $750 \mathrm{~nm}$ range.

Heliatek plans to fabricate solar panels by vacuum deposition

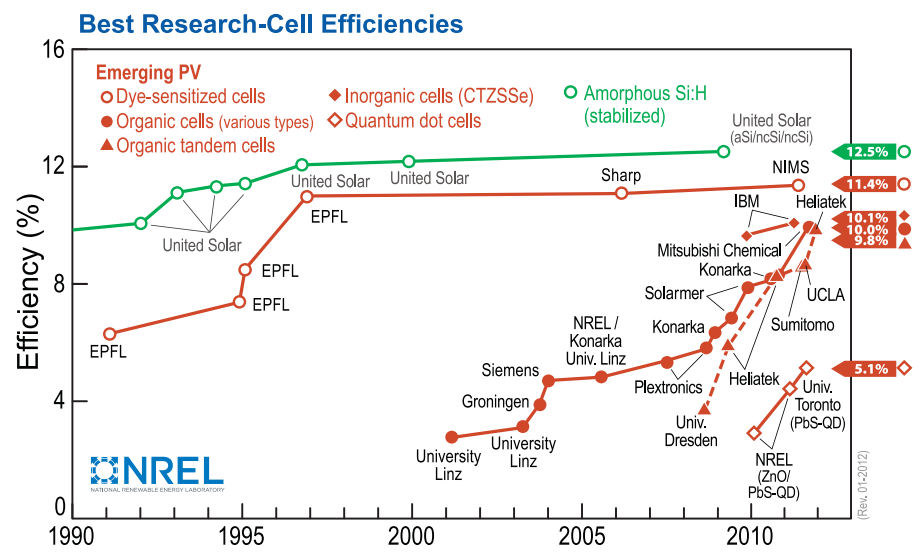

The efficiencies of organic photovoltaic solar cells have rapidly approached those of amorphous silicon and dye-sensitized solar cells within the past decade. The chart is current through December 2011. Credit: National Center for Photovoltaics, National Renewable Energy Laboratory. in a low-temperature, roll-to-roll process. Unlike spin-coating processes, it does not use a solvent, allowing for better control of crystal growth. The production speed is unlikely to exceed $2.5 \mathrm{~m} / \mathrm{min}$, whereas polymer printing processes are already much faster. "This sounds like a big disadvantage of using small molecules," said Martin Pfeiffer, Heliatek co-founder and chief technology officer. However, their cost model shows that at 1.5 $\mathrm{m} / \mathrm{min}$, the cost of the product is dominated more by material costs than by depreciation of the equipment. The competition between small-molecule and polymer OPV will be decided by "who will get better efficiency, better lifetime, and, above all, higher production yield," Pfeiffer said. Heliatek's goal is to begin mass production of OPV panels in 2012.

Molecular Solar, a spinoff company from the University of Warwick, UK, also makes small-molecule OPV. In October 2011, the company announced the creation of a solar cell with an output voltage that exceeds $4 \mathrm{~V}$. Such high-voltage cells could be used to recharge lithium-ion batteries in consumer devices.

Bazan, along with Alan Heeger and colleagues at UCSB, have created solution-processed, small-molecule solar cells, also based on a thiophene derivative. The molecule absorbs light in the optical range, especially between $600 \mathrm{~nm}$ and 800 nm. At the 2011 MRS Fall Meeting, Bazan reported achieving $7 \%$ efficiency with the material.

The most significant challenge, Bazan said, is finding the right process conditions for fabricating the molecular thin film, where the electron donors and acceptors must separate into domains of a particular size - around $30 \mathrm{~nm}$ - that nevertheless form an interconnected network. That way, the electrical charge can be carried through the material to the electrodes.

Bazan and colleagues discovered that putting a small amount — just $0.25 \%$ by volume — of an additive to the solvent resulted in a thin film with much better performance. "It's the key to achieving those record performances, but it's somewhat mysterious," he said. They described their methods in the January 2012 issue of Nature Materials. Understanding how that solvent additive works might inspire researchers to take a second look at small molecules for OPV that have been dismissed in the past.

In order for OPV to go beyond a $\$ 1 / \mathrm{W}$ fabrication cost, much work still needs to be done. "There are a lot of unexplored opportunities," Kippelen said. "At the end, there's a need for a lot of process engineering research, because it's not clear which technique will be the winner. The jury is still out there as to which is the best manufacturing technique to get to the best organic solar cells in terms of cost, efficiency, and stability."

Spitzer also emphasizes the importance of developing better barrier materials to protect OPV from oxygen, water vapor, and ultraviolet light, which tend to break down OPV and limit lifetimes.

Kippelen said that a decade ago, 10\% efficiency with OPV seemed like a pipe dream. "It was not clear if there was a path to get there," he said. "But the progress in the past few years has been very interesting." 